\title{
A tentative model for the explanation of Båth law using the order parameter of seismicity in natural time
}

\author{
Konstantina A. Papadopoulou • Efthimios S. Skordas • Nicholas V. Sarlis
}

Received: 5 September 2016/Accepted: 20 October 2016/Published online: 24 November 2016

(C) The Author(s) 2016. This article is published with open access at Springerlink.com

\begin{abstract}
Using the order parameter of seismicity defined in natural time, we suggest a simple model for the explanation of Båth law, according to which a mainshock differs in magnitude from its largest aftershock by approximately 1.2 regardless of the mainshock magnitude. In addition, the validity of Båth law is studied in the Global Centroid Moment Tensor catalogue by using two different aftershock definitions. It is found that the mean of this difference, when considering all the pairs mainshock-largest aftershock, does not markedly differ from 1.2 and the corresponding distributions do not depend on the mainshock's magnitude threshold in a statistically significant manner. Finally, the analysis of the cumulative distribution functions provides evidence in favour of the proposed model.
\end{abstract}

Keywords Båth law · Aftershocks · Order parameter . Natural time

\section{Introduction}

According to Båth law (Båth 1965; Lombardi 2002; Varotsos et al. 2011c), the magnitude difference between a mainshock and its largest aftershock is approximately 1.2

K. A. Papadopoulou · E. S. Skordas $(\bowtie) \cdot$ N. V. Sarlis Section of Solid State Physics, Department of Physics, School of Science, National and Kapodistrian University of Athens, Panepistimiopolis, Zografos, 15784 Athens, Greece

e-mail: eskordas@phys.uoa.gr

E. S. Skordas - N. V. Sarlis

Solid Earth Physics Institute, Department of Physics, School of Science, National and Kapodistrian University of Athens, Panepistimiopolis, Zografos, 15784 Athens, Greece and this value is independent of the mainshock magnitude. That means, in a catalogue of seismic events, the mean $\langle\Delta M\rangle$ of the difference between the magnitudes of each mainshock $M_{\mathrm{ms}}$ and its respective largest aftershock $M_{\mathrm{as}}^{\max }$ is given by the following relation:

$\langle\Delta M\rangle=\left\langle M_{\mathrm{ms}}-M_{\mathrm{as}}^{\max }\right\rangle=1.2$

In this paper, we first propose (in Sect. 2) a simple model in order to understand the origin of Båth law. This model makes use of the concept that the occurrence of a mainshock can be considered as a critical point and employs the order parameter proposed for seismicity on the basis of the new time frame termed natural time (Varotsos et al. 2011c) to which we now turn.

For a time-series consisting of $N$ events, the natural time can be defined (Varotsos et al. 2001, 2002, 2011c) as $\chi_{k}=$ $k / N$ and it serves as an index for the occurrence of the $k$-th event of energy $Q_{k}$. In natural time analysis, we study the evolution of the pair $\left(\chi_{k}, Q_{k}\right)$ or equivalently of the pair $\left(\chi_{k}, p_{k}\right)$ where $p_{k}=Q_{k} / \sum_{n=1}^{N} Q_{n}$. Focusing on the analysis of seismicity, the evolution of the pair $\left(\chi_{k}, M_{0 k}\right)$ is considered (Varotsos et al. 2004; Sarlis et al. 2010a), where $M_{0 k}$ denotes the seismic moment of the $k$-th event since it is a measure of its energy. The normalized power spectrum was introduced (Varotsos et al. 2001, 2002) as $\Pi(\omega)=|\Phi(\omega)|^{2}$, where

$\Phi(\omega)=\sum_{k=1}^{N} p_{k} \exp \left(\mathrm{i} \omega \frac{k}{N}\right)$

and $p_{k}=M_{0 k} / \sum_{n=1}^{N} M_{0 n}$. The Taylor expansion of $\Pi(\omega)$ leads to:

$\Pi(\omega)=1-\kappa_{1} \omega^{2}+\kappa_{2} \omega^{4}+\cdots$

where (Varotsos et al. 2001) $\kappa_{1}$ is the variance of $\chi$ : 
$\kappa_{1}=\sum_{k=1}^{N} \chi_{k}^{2} p_{k}-\left(\sum_{k=1}^{N} \chi_{k} p_{k}\right)^{2}=\left\langle\chi^{2}\right\rangle-\langle\chi\rangle^{2}$

and $\langle f(\chi)\rangle \equiv \sum_{k=1}^{N} p_{k} f\left(\chi_{k}\right)$, where $f(\chi)$ is a function of the real variable $\chi$ (Varotsos et al. 2007, 2008), e.g. when $f(\chi)=\chi^{2}$ we have $\langle f(\chi)\rangle=\left\langle\chi^{2}\right\rangle=\sum_{k=1}^{N} \chi_{k}^{2} p_{k}$. It has been shown (Varotsos et al. 2011b) that $\kappa_{1}$ becomes equal to 0.07 at the critical state for a variety of dynamical systems. In particular, for the seismicity $\kappa_{1}$ converges to 0.07 a few days before a mainshock (Varotsos et al. 2001; Sarlis et al. 2008) when starting the computation of the $\kappa_{1}$ values after the initiation of seismic electric signals (SES) activities (Varotsos and Lazaridou 1991), i.e. natural time is set to 0 at the initiation of the SES activity. SES are the transient changes of the electric field of the Earth generated by means of a mechanism of point defects (Varotsos et al. 1993; Varotsos 2008) and that have long been successfully used for short-term earthquake prediction (Varotsos et al. 2008, 2009). In this computation, only the earthquakes that occur in the area of the forthcoming earthquake, which is determined on the basis of SES properties (Varotsos and Alexopoulos 1984a, b) are considered. At the time of the mainshock, $\kappa_{1}$ tends to 0 and for this reason, $\kappa_{1}$ was proposed as an order parameter for seismicity (Varotsos et al. 2011c; Sarlis et al. 2008, 2010b; Varotsos et al. 2005, 2010, 2011a, 2013; Sarlis et al. 2013; Flores-Márquez et al. 2014).

Additionally, we examine the validity of Båth law for the events in the Global Centroid Moment Tensor (CMT) catalogue (Dziewonski et al. 1981; Ekström et al. 2012) for the time period 1/1/1976-31/12/2013 (available online on http://www.ldeo.columbia.edu/ gcmt/projects/CMT/cata log/jan76_dec13.ndk). The seismic moment $M_{0}$ which is available in the Global CMT catalogue is used (In detail, we utilize the first two columns of the fourth line for each of the catalogue's records for the moment exponent and the columns 50-56 of every fifth line for the scalar seismic moment mantissa. For example, if the exponent has the value 24 , the moment values that follow, expressed in dyn $\mathrm{cm}$, are multiplied by $10^{24}$ ). Two methods are used for the selection of the aftershocks as described in Sect. 3 and both of them show a behaviour which is compatible with the model proposed in Sect. 2. The latter discussion is made in Sect. 4. Finally, the conclusions are presented in Sect. 5.

\section{A model for the explanation of Båth law}

Here, we propose a simple model in order to understand the origin of Båth law by making use of the fact that, as already stated in Sect. 1, the variance of natural time designated as $\kappa_{1}$ may serve as an order parameter for seismicity. In addition, we consider that once $N$ events have been observed, the ratio $Q_{k} / \sum_{n=1}^{N} Q_{n}\left(=p_{k}\right)$ can be considered as a probability for the occurrence (observation) of the $k$-th event, as it has been discussed in detail in Varotsos et al. (2016) in the light of authoritative aspects of Max Planck as far as the meaning of the energy is concerned. We note that upon the occurrence of an additional event, the value of $\chi_{k}$ changes from $k / N$ to $k /(N+1)$ together with the change of $p_{k}$ from $Q_{k} / \sum_{n=1}^{N} Q_{n}$ to $Q_{k} / \sum_{n=1}^{N+1} Q_{n}$ thus leading to a change of $\kappa_{1}$ as well.

We employ the model, depicted in Fig. 1, in which most of the events in the sequence foreshocks-mainshockaftershocks have very small energies. Thus, the probabilities $p_{k}$ of occurring are almost zero apart from those in the positions $k_{1}=l+1$ considered as the mainshock with probability $p_{1}$ and $k_{2}=l+m+2$ considered as the largest magnitude aftershock detected so far with probability $p_{2}=$ $1-p_{1}$ so as $p_{1}+p_{2}=1$.

By inserting in Eq. (4) all $p_{k}=0$ apart from those of the $\chi_{k_{1}}=(l+1) / N$ and $\chi_{k_{2}}=(l+m+2) / N$ events with probabilities $p_{1}, p_{2}$, respectively, we obtain

$\kappa_{1}=p_{1} p_{2}\left(\frac{m+1}{N}\right)^{2}$

where $N$ is the length of the sequence, and $(m+1) / N=$ $[(l+m+2)-(l+1)] / N=\Delta \chi$ is the distance of the two prominent events in natural time, so that

$\kappa_{1}=p_{1} p_{2}(\Delta \chi)^{2}$.

We now set $p_{1}=Q_{1} / \sum_{n=1}^{2} Q_{n}$ and $p_{2}=Q_{2} / \sum_{n=1}^{2} Q_{n}$ and $Q_{n}$, which is proportional to the energy the earthquake produced (Kanamori 1978), is given by

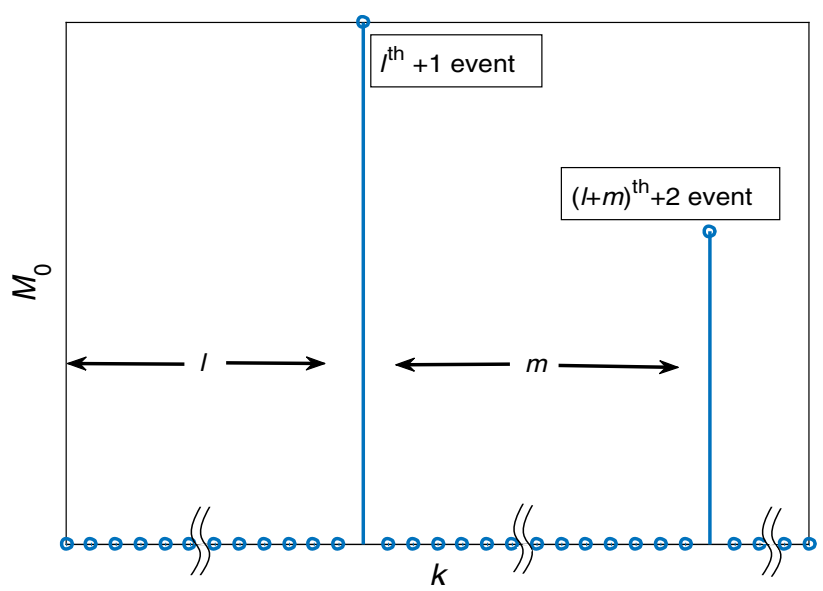

Fig. 1 The model proposed in order to understand the origin of Båth law using the order parameter of seismicity $\kappa_{1}$. Time is set to 0 at the initiation of the SES activity 
$Q_{n}=10^{1.5 M_{n}}$

where $M_{n}$ is the event's moment magnitude. The following relation is finally obtained:

$\kappa_{1}=\frac{1}{\left(1+10^{1.5 \Delta M}\right)} \frac{1}{\left(1+10^{-1.5 \Delta M}\right)}(\Delta \chi)^{2}$.

Figure 2 depicts in three dimensions $\kappa_{1}$ in relation to the difference in magnitudes $\Delta M$ of the two events mainshocklargest aftershock detected so far and their difference in natural time $\Delta \chi$. Setting $\kappa_{1}$ to its critical value, i.e. $\kappa_{1}=0.07$, we plot $\Delta \chi$ versus $\Delta M$ in Fig. 3 . We observe that $\kappa_{1}$ may reach the value of 0.07 , and thus a largest aftershock than the one already detected is expected to occur, when $|\Delta M| \leq \Delta M_{1}$ and for $\Delta \chi$ values larger than 0.5 . The value of $\Delta M_{1}$,i.e. the maximum allowed $|\Delta M|$ in order to expect a stronger earthquake, as shown in Fig. 3 can be found from Eq. (8) for $\Delta \chi=1$ and $\kappa_{1}=0.07$ which results in $\Delta M_{1} \approx 0.724$. Moreover, Fig. 2 shows that $\kappa_{1}$ is a monotonic function of $|\Delta M|$, a fact which means that the condition $|\Delta M| \leq 0.724$ is unique. The mathematical proof is provided in Appendix 1.

Now we come to the possible explanation this model provides for the origin of Båth law. If we consider the first event as the mainshock, as already stated, the result of the previous paragraph shows that the second event (largest magnitude aftershock) has to have $\Delta M>0.724$ so that the fulfilment of the condition $\kappa_{1}=0.07$ is impossible. Thus, the picture in natural time is compatible with a mainshocklargest aftershock pair and no stronger earthquake is expected to take place "close" to the mainshock. On the other hand, if $\Delta M \leq 0.724$ there is a probability that $\kappa_{1}$ may reach the value 0.07 and a stronger earthquake will occur. In this case, the original picture mainshock-largest aftershock pair is falsified by the true events and hence this pair of events is not included in the calculation of $\langle\Delta M\rangle$ of Eq. (1). Of course, there is also a possibility that $\kappa_{1}$ may

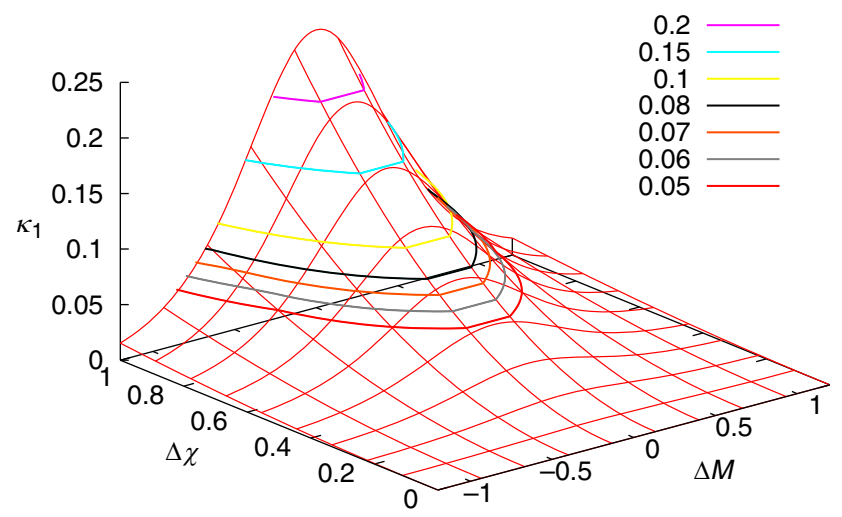

Fig. 2 The order parameter of seismicity $\kappa_{1}$ in relation to the difference in magnitudes $\Delta M$ of the two events under consideration and their difference in natural time $\Delta \chi$

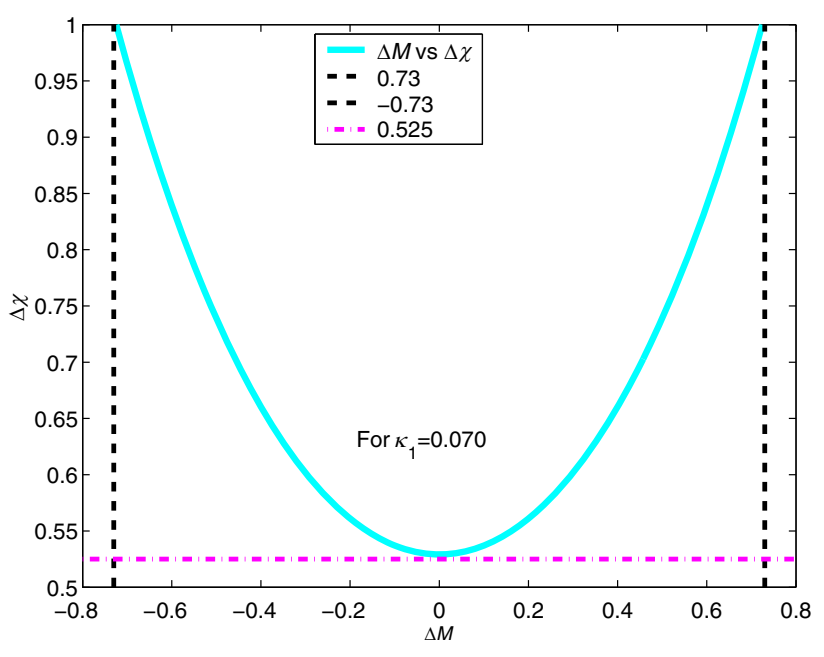

Fig. $3 \Delta M$ in relation with $\Delta \chi$ in the critical state where $\kappa_{1}=0.070$

not reach 0.07 as long as the activity "close" to the mainshock is in progress but at a later time, thus this pair of events contributes to the aforementioned average value with $\Delta M \leq 0.724$. Such cases, however, are expected to be fewer than those with $\Delta M>0.724$ since in the latter it is impossible to have a stronger earthquake than the two events detected so far. Thus, we expect that the average value of $\Delta M$ should be larger than 0.724 as suggested by Båth law.

Specifically, if we assume a piecewise constant probability distribution for $\Delta M$ of the form

$$
\begin{aligned}
p(\Delta M)= & p_{0}\left[\Theta(\Delta M) \Theta\left(\Delta M_{1}-\Delta M\right)\right. \\
& \left.+\lambda \Theta\left(\Delta M-\Delta M_{1}\right) \Theta\left(2 \Delta M_{1}-\Delta M\right)\right],
\end{aligned}
$$

where $\Theta(x)$ is the Heaviside unit step function, i.e. $\Theta(x)=$ 1 for $x \geq 0$ and 0 otherwise, which is drawn with the red solid line in Fig. 4, we practically assume that the probability to observe $\Delta M>\Delta M_{1}$ is $\lambda$ times larger than that of observing $\Delta M \leq \Delta M_{1}$. Using Eq. (9), we obtain (see Appendix 2) that the average value of $\Delta M$ is given by

$\langle\Delta M\rangle=\frac{\Delta M_{1}}{2} \frac{(1+3 \lambda)}{(1+\lambda)}$,

which for $\lambda \rightarrow \infty$ results in $\langle\Delta M\rangle \approx 1.086$, i.e. a value very close to that of Båth law in Eq. (1). We note that Zaliapin and Ben-Zion (Zaliapin and Ben-Zion 2013a) have observed that for earthquake sequences in southern California $\Delta M$ follows an almost uniform distribution in the range $[0,2]$ with an exponential tail for larger values of $\Delta M$, thus supporting the value $\langle\Delta M\rangle=1.1$ which has been found (Zaliapin and Ben-Zion 2013a) in the observed seismicity of southern California. They have also identified (Zaliapin and Ben-Zion 2013b) that there exist two basic types of earthquake clustering: the burst-like sequences which are consistent with highly brittle behaviour and the 


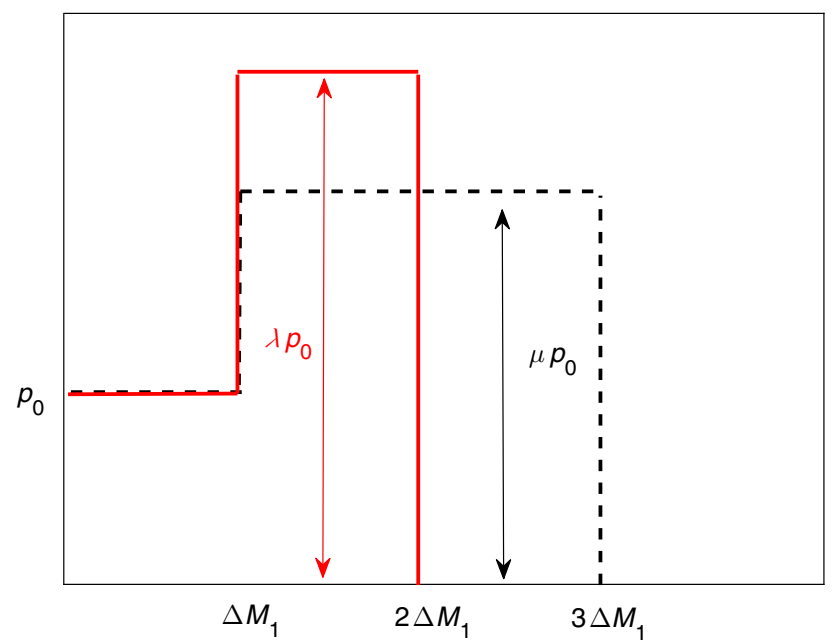

Fig. 4 The piecewise constant probability distribution assumed for $\Delta M$ (red solid line for Eq. (9) and black-dashed line for Eq. (11)) where the probability to observe $\Delta M>\Delta M_{1}$ is larger than that of observing $\Delta M \leq \Delta M_{1}$

swarm-like sequences consistent with mixed brittle-ductile behaviour. The latter sequences result in smaller $\Delta M$ values than those of the former. The suggested model is not inconsistent with this finding since it differentiates between small $\left(\Delta M<\Delta M_{1}\right)$ and large $\Delta M$ classes of aftershock sequences.

If we further extend our model to allow $\Delta M$ to be larger than $2 \Delta M_{1}$, which is closer to the real case as we will see in the next Section, we may consider the distribution

$$
\begin{aligned}
p(\Delta M)= & p_{0}\left[\Theta(\Delta M) \Theta\left(\Delta M_{1}-\Delta M\right)\right. \\
& \left.+\mu \Theta\left(\Delta M-\Delta M_{1}\right) \Theta\left(3 \Delta M_{1}-\Delta M\right)\right],
\end{aligned}
$$

which is drawn with the black-dashed line in Fig. 4. Equation (11) results in (see Appendix 3)

$\langle\Delta M\rangle=\frac{\Delta M_{1}}{2} \frac{(1+8 \mu)}{(1+2 \mu)}$.

If we substitute in the latter expression values of $\mu$ which are close to the experimental ones, we can obtain a value for Båth law close to 1.2 as in Eq. (1). This will be shown in Sect. 4.

\section{Båth law in the Global CMT catalogue: the procedures followed and the results}

For the calculation of $\Delta M$, we adopt as earthquake magnitude the moment magnitude $M_{w}$ which results from the seismic moment $M_{o}$ through the relation (Kanamori 1978)

$M_{\mathrm{w}}=\frac{2}{3} \log _{10} M_{0}-10.7$, where $M_{0}$ is measured in dyn $\cdot \mathrm{cm}\left(10^{-7} \mathrm{~N} \cdot \mathrm{m}\right)$. The minimal reported magnitude results in 4.6 (cf. a single 4.3 earthquake is reported in the Global CMT catalogue). The completeness magnitude threshold $M_{\mathrm{c}}$ lies within 5.3-5.4 for the period before 2004 and decreases to 5.0 during the period 2004-2010 (Ekström et al. 2012). Two methods for the identification of the mainshock and its largest aftershock were used.

\subsection{First method}

In this method, we follow the definitions suggested in Shcherbakov et al. (2004): For each mainshock, we consider as aftershocks all earthquakes that took place within a space-time window. The time span of this window is 1 year (Shcherbakov et al. 2013; Zakharova et al. 2013) after the mainshock and the spatial dimensions are those of a rectangular region of side "length" of $d\left(L / L_{1}\right)$ degrees centred around the mainshock. We consider (Shcherbakov et al. 2004)

$L=0.02 \times 10^{0.5 M_{\mathrm{ms}}}$

and $L_{1}=111 \mathrm{~km}$ is the length per degree. This value of $L_{1}$ corresponds to the length of one degree either in the NorthSouth or East-West direction at the equator.

For each earthquake in the catalogue a space-time window as described above was created around it. If this earthquake was not the largest event inside its respective window, then it was rejected as a mainshock, otherwise it was considered as a mainshock. The largest aftershock of each sequence was then designated as the second maximum magnitude inside each mainshock's window and a total of 3100 pairs of mainshock-largest aftershock were identified.

We now compute the mean value $\langle\Delta M\rangle=\left\langle M_{\mathrm{ms}}-\right.$ $\left.M_{\mathrm{as}}^{\max }\right\rangle$ and the standard deviation $\sigma$ of $\Delta M$ for various mainshock magnitude thresholds, i.e. first we compute $\sigma$ and $\langle\Delta M\rangle$ for $M_{\mathrm{ms}} \geq 5\left(\equiv M_{\mathrm{ms}, \text { thres }}\right)$ then for $M_{\mathrm{ms}} \geq 5.1$ and so on. Figure 5 depicts $\langle\Delta M\rangle=\left\langle M_{\mathrm{ms}}-M_{\mathrm{as}}^{\max }\right\rangle$ and $\sigma$ versus the $M_{\mathrm{ms}, \text { thres }}$ and the number of mainshocks. We find that for $M_{\mathrm{ms} \text {.thres }} \geq 5.7$ the value of $\langle\Delta M\rangle$ does not differ significantly from 1.2 (see the yellow region in Fig. 5 which has been drawn as a guide to the eye in view of the large observed $\sigma$ values) at least for the cases where the statistics come from more than 30 mainshocks, i.e. for $M_{\text {ms,thres }}$ up to 7.9. The linear trend that appears is certainly related to the relatively high completeness magnitude. We verified this fact by discarding all earthquakes with $M<M_{c}=5.3$ and repeating the computations. By comparing the new $\langle\Delta M\rangle$ curve with the existing one, we observed almost the same linear trend and a horizontal displacement. The two curves result in the same values for 


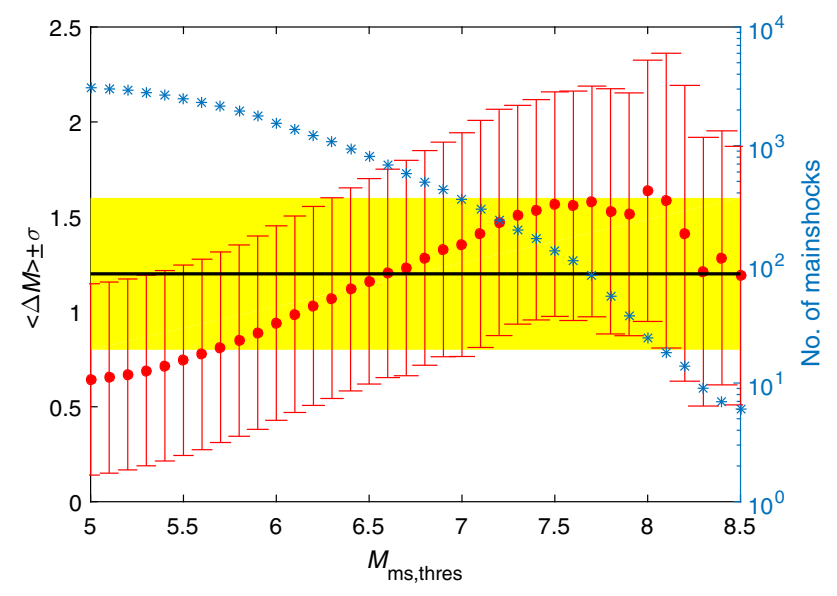

Fig. $5\langle\Delta M\rangle=\left\langle M_{\mathrm{ms}}-M_{\mathrm{as}}^{\max }\right\rangle$ and $\pm \sigma$ versus the $M_{\mathrm{ms} \text {,thres }}$ (red-filled circles, left scale) and the number of mainshocks (blue stars, right scale) used for the first method of mainshock-largest aftershock identification where a rectangular region is considered around the mainshock. A black bold horizontal line is drawn for Båth law, i.e. $\langle\Delta M\rangle=1.2$. The yellow area defines the region $\langle\Delta M\rangle=1.2 \pm 0.4$

$M_{\mathrm{ms}, \text { thres }} \geq 7.9$. For $M_{\mathrm{ms} \text {,thres }}$ around 8.3 and above (where the number of events is less than 10) the results are not credible because only a few events with large magnitudes have occurred (see Fig. 5). Table 1 shows the corresponding values of $\langle\Delta M\rangle$ and $\sigma$ for various $M_{\mathrm{ms} \text {, thres }}$ values alongside their standard errors (Sarlis and Christopoulos 2012; Ahn and Fessler 2003) $\delta\langle\Delta M\rangle=\sigma / \sqrt{N}$ and $\delta \sigma=\sigma / \sqrt{2(N-1)}$.

In order to examine whether the $\Delta M$ distributions corresponding to different $M_{\mathrm{ms}, \text { thres }}$ can be statistically discriminated in practice, we generated $10^{3}$ samples of five $\Delta M$ values coming from the distribution for $M_{\mathrm{ms}, \text { thres }}=5.7$ (assumed Gaussian) and compared them to another five $\Delta M$ values coming from the distribution for $M_{\mathrm{ms} \text {,thres }}=7.7$ (also assumed Gaussian) by using the $t$ test for the

Table 1 Indicatively, $\langle\Delta M\rangle=\left\langle M_{\mathrm{ms}}-M_{\mathrm{as}}^{\max }\right\rangle, \sigma$ and number $N$ of mainshock-aftershock pairs used in the computations for various values of $M_{\mathrm{ms} \text {,thres }}$ for the first method of mainshock-largest aftershock identification where a rectangular region is considered around the mainshock

\begin{tabular}{lccr}
\hline$M_{\text {ms,thres }}$ & $\langle\Delta M\rangle \pm \delta\langle\Delta M\rangle$ & $\sigma \pm \delta \sigma$ & $N$ \\
\hline 5.0 & $0.644 \pm 0.009$ & $0.504 \pm 0.006$ & 3056 \\
5.5 & $0.744 \pm 0.010$ & $0.501 \pm 0.007$ & 2492 \\
6.0 & $0.941 \pm 0.013$ & $0.512 \pm 0.009$ & 1557 \\
6.5 & $1.161 \pm 0.019$ & $0.541 \pm 0.014$ & 807 \\
6.6 & $1.202 \pm 0.021$ & $0.549 \pm 0.015$ & 695 \\
7.0 & $1.35 \pm 0.03$ & $0.589 \pm 0.022$ & 353 \\
7.5 & $1.57 \pm 0.05$ & $0.59 \pm 0.04$ & 131 \\
8.0 & $1.64 \pm 0.14$ & $0.69 \pm 0.10$ & 24 \\
\hline
\end{tabular}

difference of the means of the two samples. This study revealed that there is on average a $15 \%$ probability that the two samples come from distributions with the same mean. This reveals that although $\langle\Delta M\rangle$ may differ, the corresponding distributions do not depend on the mainshock's magnitude threshold in a statistically significant manner.

\subsection{Second method}

In this case, we consider the definition of aftershocks suggested in Zaliapin et al. (2008) which uses the nearestneighbour distance: For each earthquake $i$ in a catalogue, we consider its occurrence time $t_{i}$, latitude $\varphi_{i}$ (in radians), longitude $\theta_{i}$ (in radians) and magnitude $m_{i}$. For a pair of earthquakes $i$ and $j$, the time-space-magnitude distance is defined (Baiesi and Paczuski 2004) as

$n_{i j}= \begin{cases}t_{i j}\left(r_{i j}\right)^{d} 10^{-b m_{i}}, & t_{i j}>0 \\ \infty, & t_{i j}<0\end{cases}$

where $t_{i j}=t_{j}-t_{i}$ is the interoccurrence time in years, $d$ is the fractal dimension of the epicentres, $b$ the GutenbergRichter parameter and $r_{i j} \geq 0$ is the spatial distance between the epicentres (Batac and Kantz 2014; Baiesi and Paczuski 2004):

$r_{i j}=R_{\mathrm{E}} \arccos \left(\sin \varphi_{i} \sin \varphi_{j}+\cos \varphi_{i} \cos \varphi_{j} \cos \left(\theta_{j}-\theta_{i}\right)\right)$

where $R_{\mathrm{E}}=6371 \mathrm{~km}$, the Earth's radius.

We studied the Global CMT catalogue following the method suggested in Zaliapin and Ben-Zion (2013a, b, 2015) for the identification of earthquake clusters: For each earthquake $j$, going backwards in time, we find its nearest-neighbour $i$ and their corresponding distance $n_{i j}$. The nearest-neighbour, which is called the parent, can be the parent of multiple events which are called offsprings. We should note that each possible parent-offspring pair is assigned a corresponding distance $n$ but the nearestneighbour is defined as the pair with minimum $n_{i j}$ (Zaliapin et al. 2008; Zaliapin and Ben-Zion 2013a). Normalizing the time and space distances by the magnitude of the parent, we have (Zaliapin et al. 2008; Zaliapin and Ben-Zion 2015)

$T_{i j}=t_{i j} 10^{-q b m_{i}} ; R_{i j}=\left(r_{i j}\right)^{d} 10^{-p b m_{i}} ; q+p=1$

where $T$ is referred to (Zaliapin et al. 2008; Zaliapin and Ben-Zion 2015) as the rescaled time and $R$ as the rescaled distance. We selected $b=1, d=1.6$ and $p=0.5$ (Zaliapin et al. 2008; Zaliapin and Ben-Zion 2015).

As it was shown in Zaliapin and Ben-Zion (2013a), Zaliapin et al. (2008) and Zaliapin and Ben-Zion (2015), earthquake catalogues show a bimodal joint distribution of $\left(\log _{10} T, \log _{10} R\right)$. One mode corresponds to the background events-like events in a Poisson process-while 
the other corresponds to the clustered events with $n_{i j}<10^{-5}$, i.e. events closer in time and space to their parents than expected in a Poisson process (Zaliapin and Ben-Zion 2013a, 2015; Zaliapin et al. 2008). Figure 6 depicts this bimodal distribution for the Global CMT catalogue. By discarding all $n_{i j}>10^{-5}$, the events in a catalogue are divided into single events and families that contain multiple events connected to their parents with nearest-neighbour distances $n_{i j}<10^{-5}$, i.e. strong links (Zaliapin and Ben-Zion 2013a). The largest event in a family is considered to be the mainshock. The events before it are considered as foreshocks, while the events after it are considered to be the aftershocks (Zaliapin and Ben-Zion 2013a). Analysing Global CMT catalogue by employing this technique, 3144 pairs of mainshock-largest aftershock were extracted, i.e. 3144 families.

In order to study Båth law, we keep our computations to these families and Fig. 7 depicts $\langle\Delta M\rangle=\left\langle M_{\mathrm{ms}}-M_{\mathrm{as}}^{\max }\right\rangle$ and $\sigma$ versus the mainshock threshold $M_{\mathrm{ms}, \text { thres }}$ and the number of mainshocks used in the calculation. Table 2 shows the corresponding values of $\langle\Delta M\rangle$ and $\sigma$ for different $M_{\text {ms,thres }}$ values alongside their standard errors (Sarlis and Christopoulos 2012; Ahn and Fessler 2003).

Here, we also find that for $M_{\mathrm{ms}, \text { thres }} \geq 5.8$ the value of $\langle\Delta M\rangle$ does not differ significantly from 1.2 at least for the cases where the statistics come from more than 10 mainshocks, i.e. for $M_{\mathrm{ms}, \text { thres }}$ up to 8.2 (see the yellow region in Fig. 7). There is again the linear trend but we have to note that the results of this method lead to the existence of a clear plateau in $\langle\Delta M\rangle$ (see Fig. 7 for $M_{\text {ms,thres }} \in[6.8,8.1]$ )

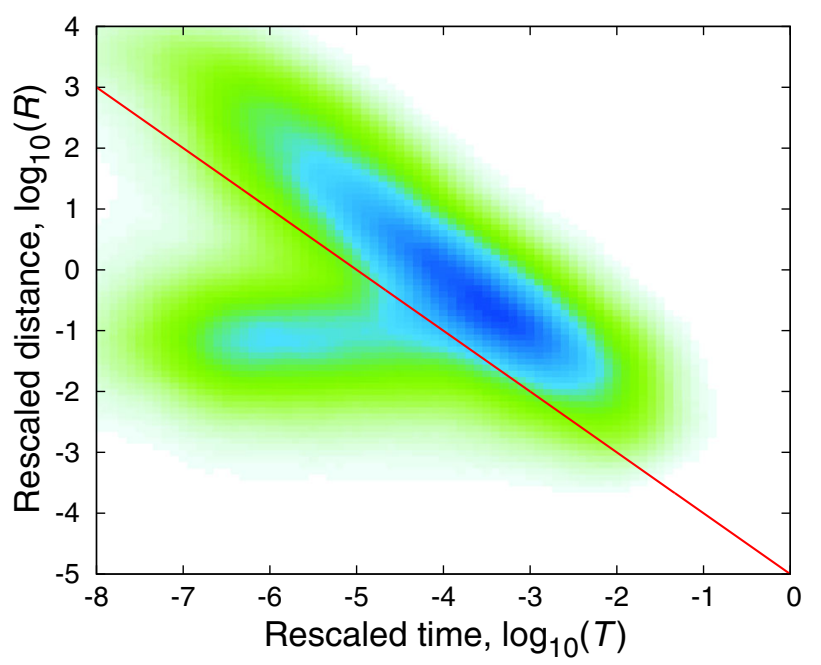

Fig. 6 The joint probability density of the rescaled components $\left(\log _{10} T, \log _{10} R\right)$ of the earthquake nearest-neighbour distance for the Global CMT catalogue. The darker colour corresponds to larger density. The upper right mode is the background mode while the left bottom mode is the clustered mode (see text).The red solid line $\log _{10} T+\log _{10} R=-5$ is also drawn

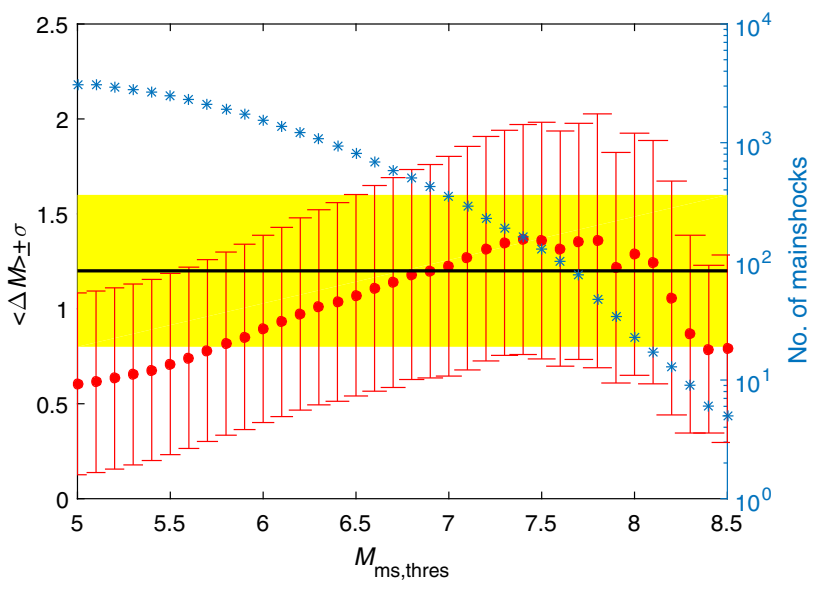

Fig. $7\langle\Delta M\rangle=\left\langle M_{\mathrm{ms}}-M_{\mathrm{as}}^{\max }\right\rangle$ and $\pm \sigma$ versus the $M_{\mathrm{ms} \text {,thres }}$ (red-filled circles, left scale) and the number of mainshocks (blue stars, right scale) used for the second method of mainshock-largest aftershock identification where for each earthquake in the Global CMT catalogue we identify its nearest-neighbour. A black thick horizontal line is drawn for Båth law, i.e. $\langle\Delta M\rangle=1.2$. The yellow area defines the region $\langle\Delta M\rangle=1.2 \pm 0.4$

Table 2 Indicatively, $\langle\Delta M\rangle=\left\langle M_{\mathrm{ms}}-M_{\mathrm{as}}^{\max }\right\rangle, \sigma$ and number $N$ of mainshock-aftershock pairs used in the computations for various values of $M_{\mathrm{ms} \text {,thres }}$ for the second method of mainshock-largest aftershock identification where for each earthquake in the Global CMT catalogue, we identify its nearest-neighbour

\begin{tabular}{lccr}
\hline$M_{\text {ms,thres }}$ & $\langle\Delta M\rangle \pm \delta\langle\Delta M\rangle$ & $\sigma \pm \delta \sigma$ & $N$ \\
\hline 5.0 & $0.605 \pm 0.009$ & $0.479 \pm 0.006$ & 3115 \\
5.5 & $0.710 \pm 0.010$ & $0.477 \pm 0.007$ & 2489 \\
6.0 & $0.894 \pm 0.013$ & $0.493 \pm 0.009$ & 1540 \\
6.5 & $1.071 \pm 0.018$ & $0.530 \pm 0.013$ & 809 \\
6.9 & $1.20 \pm 0.03$ & $0.560 \pm 0.020$ & 427 \\
7.0 & $1.22 \pm 0.03$ & $0.579 \pm 0.022$ & 350 \\
7.5 & $1.36 \pm 0.06$ & $0.62 \pm 0.04$ & 127 \\
8.0 & $1.29 \pm 0.13$ & $0.64 \pm 0.09$ & 23 \\
\hline
\end{tabular}

where $\langle\Delta M\rangle$ varies with a standard deviation 0.06 around the value 1.29, very close to that of the Båth law of Eq. (1) (cf. a similar calculation for the first method for $M_{\mathrm{ms}, \text { thres }} \in$ $[7.1,8.2]$ leads to 1.53 with standard deviation 0.07$)$. In our opinion this result is not fortuitous, but is due to the fact that in the second method the aftershocks are selected according to the clustering properties of seismicity, e.g. Eq. (15) and Fig. 6.

\section{Comparison of the experimental results with the model}

Let us now examine what happens for $\Delta M$ for the pairs mainshock-largest aftershock derived from the two 
methods presented in Sects. 3.1 and 3.2 for a relatively high mainshock magnitude threshold close to that $\left(M_{\mathrm{ms} \text {,thres }}=7\right)$ considered by Båth in his original paper (Båth 1965). As an example, we consider $M_{\mathrm{ms}} \geq 7.5$ which is also larger than or equal to $3 \Delta M_{1}+M_{\mathrm{c}}$ for the whole period of study (cf. $M_{\mathrm{c}} \approx 5.35$, see Sect. 3). In Figs. 8 and 9, we plot the cumulative distribution function (CDF) of $\Delta M$ for the first and the second method of aftershock identification, respectively, and model these distributions according to Eq. (11). For this reason, we perform two linear fits for each CDF by dividing the data into two intervals, one before $\Delta M_{1}$ and the other after $\Delta M_{1}$ covering the remaining ("linear") portion of the CDF up to approximately $90 \%$ so as to avoid the effect of the tails. The intervals we selected are namely $\left[0, \Delta M_{1}\right)$ and $\left(\Delta M_{1}, 3 \Delta M_{1}\right]$ and the corresponding results are shown in Figs. 8 and 9 . The ratio between the two slopes is $\mu=$ $3.08 \pm 0.19$ for the first method of aftershock identification and $\mu=2.01 \pm 0.06$ for the second method. By inserting these values for $\mu$ in Eq. (12), we obtain 1.30 and 1.23, respectively, for the value in Bath law. Thus, by inserting the experimental values into the proposed model the resulting $\langle\Delta M\rangle$ values are in accordance with the corresponding value in Båth law.

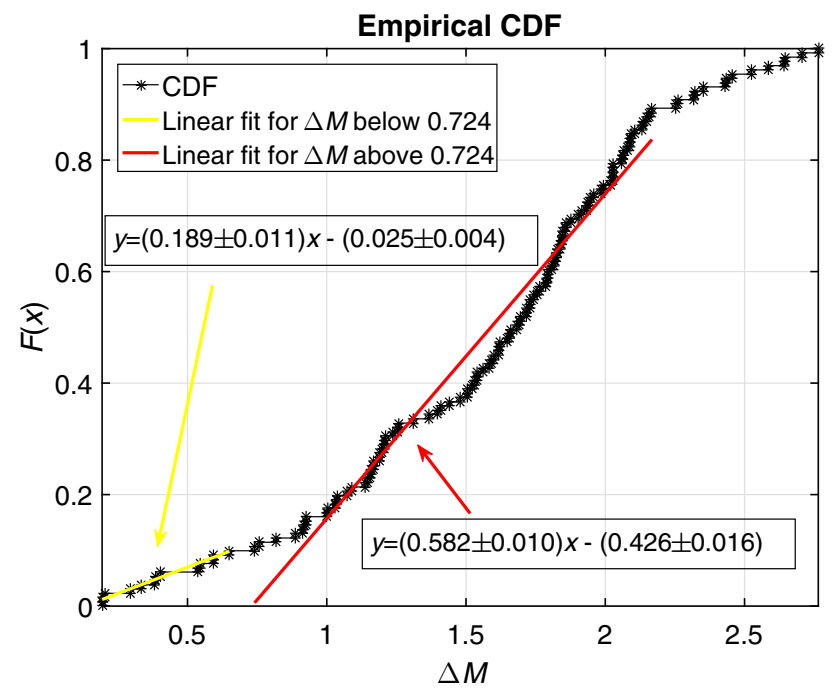

Fig. 8 Cumulative distribution function of $\Delta M$ and its two linear fits in the intervals $\left[0, \Delta M_{1}\right)$ (yellow line) and $\left(\Delta M_{1}, 3 \Delta M_{1}\right]$ (red line) for the first method of mainshock-largest aftershock identification where a rectangular region is created around the mainshock

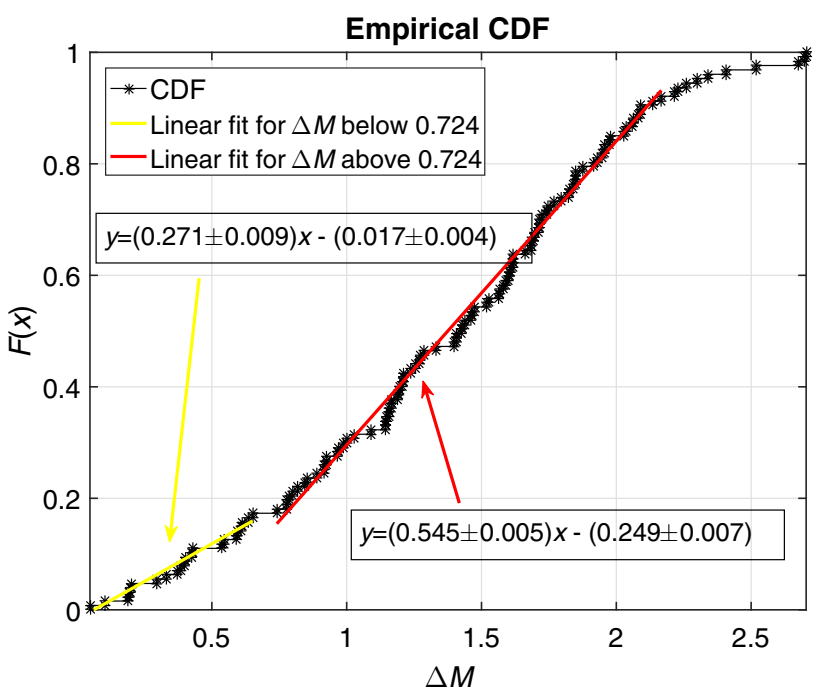

Fig. 9 Cumulative distribution function of $\Delta M$ and its two linear fits in the intervals $\left[0, \Delta M_{1}\right)$ (yellow line) and $\left(\Delta M_{1}, 3 \Delta M_{1}\right]$ (red line) for the second method of mainshock-largest aftershock identification where for each earthquake in the Global CMT catalogue we identify its nearest-neighbour

\section{Conclusions}

We examined the validity of Båth law in the Global CMT catalogue. We found that the corresponding value in Båth law does not markedly differ from 1.2 and the corresponding distributions of $\Delta M$ do not depend on the mainshock's magnitude threshold in a statistically significant manner. We note that by using the second method of aftershock identification, which makes use of the notion of earthquake families, the experimental values of $\langle\Delta M\rangle$ we derived are closer to 1.2 than those obtained by the first method that uses a space-time window around each mainshock, e.g. see Tables 1 and 2.

A simple model, utilizing the order parameter $\kappa_{1}$ of seismicity in natural time, to explain the value of 1.2 has been proposed. Briefly, we use the fact that after the initiation of the SES activity and when studying the seismicity in the area prone to suffer the large earthquake, $\kappa_{1}$ approaches the value 0.07 before the mainshock. Considering that this condition should be valid before every mainshock the model suggests that the probability to observe $\Delta M>0.724$ should be markedly larger than that of $\Delta M \leq 0.724$. Based on this, two simple probability distributions for $\Delta M$ have been considered and the related expressions for $\langle\Delta M\rangle$ have been obtained. When these expressions are compared to the experimental data and combined with the modern aspects of earthquake clustering they may lead to values of $\langle\Delta M\rangle$ close to 1.2 in accordance with the Båth law. 
Acknowledgements We are grateful to Ilya Zaliapin for providing us with the Matlab code for the nearest-neighbour identification and the extraction of the clustered events which were used in the second method of aftershock identification described in Sect. 3.

Open Access This article is distributed under the terms of the Creative Commons Attribution 4.0 International License (http://creative commons.org/licenses/by/4.0/), which permits unrestricted use, distribution, and reproduction in any medium, provided you give appropriate credit to the original author(s) and the source, provide a link to the Creative Commons license, and indicate if changes were made.

\section{Appendix 1}

Here, we show that the conditions $|\Delta M| \leq 0.724$ and $\Delta \chi>0.5$ are unique. The partial derivative of Eq. (8) with respect to $\Delta \chi$ is

$\frac{\partial \kappa_{1}}{\partial \Delta \chi}=\frac{2}{\left(1+10^{1.5 \Delta M}\right)} \frac{(\Delta \chi)}{\left(1+10^{-1.5 \Delta M}\right)}$

and since $\Delta \chi \in(0,1], \partial \kappa_{1} / \partial \Delta \chi$ is always positive which means that $\kappa_{1}$ is a monotonic function of $\Delta \chi$.

Next, we compute the partial derivative of Eq. (8) with respect to $\Delta M$. After calculations we derive

$\frac{\partial \kappa_{1}}{\partial \Delta M}=\frac{(\Delta \chi)^{2} 1.5 \ln 10\left(1-10^{3 \Delta M}\right)}{\left(1+10^{1.5 \Delta M}\right)^{2}\left(1+10^{-1.5 \Delta M}\right)^{2} 10^{1.5 \Delta M}}$

and for $a=\frac{(\Delta \chi)^{2} 1.5 \ln 10}{\left(1+10^{1.5 \Delta M}\right)^{2}\left(1+10^{-1.5 \Delta M}\right)^{2}}$, we finally obtain

$\frac{\partial \kappa_{1}}{\partial \Delta M}=a \frac{\left(1-10^{3 \Delta M}\right)}{10^{1.5 \Delta M}}$

which results in

$\frac{\partial \kappa_{1}}{\partial \Delta M}=-2 a \sinh (1.5 \Delta M \ln 10)$

where $a>0$. Since the model does not take under consideration whether the mainshock is larger than its largest aftershock or not, there are two possibilities:

(1) $\Delta M>0$ so that $\sinh (1.5 \Delta M \ln 10)>0$. In this case, $\partial \kappa_{1} / \partial \Delta M<0$.

(2) $\Delta M<0 \quad$ so that $\sinh (1.5 \Delta M \ln 10)<0$ since $\sinh (-x)=-\sinh (x)$. In this case, we have $\partial \kappa_{1} / \partial \Delta M>0$.

In either of the cases above, which are summarized in Fig. 2, $\kappa_{1}$ is a monotonic function of $|\Delta M|$, a fact which means that the condition $|\Delta M| \leq 0.724$ is unique.

\section{Appendix 2}

By normalizing the probability of observing $\Delta M$, i.e. demanding the area under the red solid line in Fig. 4 to be unity, we have $p_{0} \Delta M_{1}+\lambda p_{0} \Delta M_{1}=1$

and solving for $p_{0}$ we derive

$p_{0}=\frac{1}{\Delta M_{1}(1+\lambda)}$.

The average value of $\Delta M$ is given by

$\langle\Delta M\rangle=\int_{0}^{\Delta M_{1}} M p_{0} \mathrm{~d} M+\int_{\Delta M_{1}}^{2 \Delta M_{1}} \lambda M p_{0} \mathrm{~d} M$

and by substituting Eq. (23) for $p_{0}$ we finally obtain

$\langle\Delta M\rangle=\frac{\Delta M_{1}}{2} \frac{(1+3 \lambda)}{(1+\lambda)}$.

For $\lambda \rightarrow \infty$, the last equation gives

$\langle\Delta M\rangle=\frac{3}{2} \Delta M_{1}$,

which for $\Delta M_{1}=0.724$ results in $\langle\Delta M\rangle=1.086$ as stated in the main text.

\section{Appendix 3}

By normalizing the probability of observing $\Delta M$, i.e. demanding the area under the black-dashed line in Fig. 4 to be unity, we have

$p_{0} \Delta M_{1}+2 \mu p_{0} \Delta M_{1}=1$

and solving for $p_{0}$ we find

$p_{0}=\frac{1}{\Delta M_{1}(1+2 \mu)}$.

The average value of $\Delta M$ is given by

$\langle\Delta M\rangle=\int_{0}^{\Delta M_{1}} M p_{0} \mathrm{~d} M+\int_{\Delta M_{1}}^{3 \Delta M_{1}} \mu M p_{0} \mathrm{~d} M$

and by substituting Eq. (28) for $p_{0}$ we finally obtain

$\langle\Delta M\rangle=\frac{\Delta M_{1}}{2} \frac{(1+8 \mu)}{(1+2 \mu)}$,

which is just Eq. (12) of the main text.

\section{References}

Ahn S, Fessler A (2003) Standard errors of mean, variance, and standard deviation estimators. http://www.eecs.umich.edu/ fess ler/papers/lists/files/tr/stderr.pdf, technical Report. Ann Arbor, MI, USA: Comm. and Sign. Proc. Lab., Dept. of EECS, Univ. of Michigan, July 2003

Baiesi M, Paczuski M (2004) Scale-free networks of earthquakes and aftershocks. Phys Rev E 69:066106

Batac R, Kantz H (2014) Observing spatio-temporal clustering and separation using interevent distributions of regional earthquakes. Nonlin Process Geophys 21:735-744 
Båth M (1965) Lateral inhomogeneities of the upper mantle. Tectonophysics 2(6):483-514

Dziewonski A, Chou TA, Woodhouse J (1981) Determination of earthquake source parameters from waveform data for studies of global and regional seismicity. J Geophys Res Solid Earth (1978-2012) 86(B4):2825-2852

Ekström G, Nettles M, Dziewonski A (2012) The global cmt project 2004-2010: centroid-moment tensors for 13,017 earthquakes. Phys Earth Planet Inter 200:1-9

Flores-Márquez E, Vargas C, Telesca L, Ramrez-Rojas A (2014) Analysis of the distribution of the order parameter of synthetic seismicity generated by a simple spring-block system with asperities. Phys A 393:508-512

Kanamori H (1978) Quantification of earthquakes. Nature 271:411-414

Lombardi AM (2002) Probabilistic interpretation of Båth's Law. Ann Geophys 45(3-4):455-472

Sarlis NV, Christopoulos SRG (2012) Natural time analysis of the centennial earthquake catalog. Chaos 22:023123

Sarlis NV, Skordas ES, Lazaridou MS, Varotsos PA (2008) Investigation of seismicity after the initiation of a seismic electric signal activity until the main shock. Proc Jpn Acad Ser B 84:331-343

Sarlis NV, Skordas ES, Varotsos PA (2010a) Nonextensivity and natural time: the case of seismicity. Phys Rev E 82:021110

Sarlis NV, Skordas ES, Varotsos PA (2010b) Order parameter fluctuations of seismicity in natural time before and after mainshocks. EPL 91:59001

Sarlis NV, Skordas ES, Varotsos PA, Nagao T, Kamogawa M, Tanaka H, Uyeda S (2013) Minimum of the order parameter fluctuations of seismicity before major earthquakes in Japan. Proc Natl Acad Sci USA 110:13734-13738

Shcherbakov R, Turcotte DL, Rundle JB (2004) A generalized Omori's law for earthquake aftershock decay. Geophys Res Lett 31:L11613

Shcherbakov R, Goda K, Ivanian A, Atkinson GM (2013) Aftershock statistics of major subduction earthquakes. Bull Seismol Soc Am 103:3222-3234

Varotsos $\mathrm{P}$ (2008) Point defect parameters in $\beta-\mathrm{PbF}_{2}$ revisited. Solid State Ion 179:438-441

Varotsos P, Alexopoulos K (1984a) Physical properties of the variations of the electric field of the earth preceding earthquakes, I. Tectonophysics 110:73-98

Varotsos P, Alexopoulos K (1984b) Physical properties of the variations of the electric field of the earth preceding earthquakes, II. Tectonophysics 110:99-125

Varotsos P, Lazaridou M (1991) Latest aspects of earthquake prediction in Greece based on seismic electric signals. Tectonophysics 188:321-347

Varotsos P, Alexopoulos K, Lazaridou M (1993) Latest aspects of earthquake prediction in Greece based on seismic electric signals, II. Tectonophysics 224:1-37

Varotsos PA, Sarlis NV, Skordas ES (2001) Spatio-temporal complexity aspects on the interrelation between seismic electric signals and seismicity. Pract Athens Acad 76:294-321
Varotsos PA, Sarlis NV, Skordas ES (2002) Long-range correlations in the electric signals that precede rupture. Phys Rev E 66:011902

Varotsos PA, Sarlis NV, Skordas ES, Tanaka HK (2004) A plausible explanation of the $b$-value in the Gutenberg-Richter law from first Principles. Proc Jpn Acad Ser B 80:429-434

Varotsos PA, Sarlis NV, Tanaka HK, Skordas ES (2005) Similarity of fluctuations in correlated systems: the case of seismicity. Phys Rev E 72:041103

Varotsos PA, Sarlis NV, Skordas ES, Lazaridou MS (2007) Identifying sudden cardiac death risk and specifying its occurrence time by analyzing electrocardiograms in natural time. Appl Phys Lett 91:064106

Varotsos PA, Sarlis NV, Skordas ES, Lazaridou MS (2008) Fluctuations, under time reversal, of the natural time and the entropy distinguish similar looking electric signals of different dynamics. J Appl Phys 103:014906

Varotsos PA, Sarlis NV, Skordas ES (2009) Detrended fluctuation analysis of the magnetic and electric field variations that precede rupture. Chaos 19:023114

Varotsos PA, Sarlis NV, Skordas ES, Uyeda S, Kamogawa M (2010) Natural time analysis of critical phenomena. The case of Seismicity. EPL 92:29002

Varotsos P, Sarlis N, Skordas E (2011a) Scale-specific order parameter fluctuations of seismicity in natural time before mainshocks. EPL 96:59002

Varotsos P, Sarlis NV, Skordas ES, Uyeda S, Kamogawa M (2011b) Natural time analysis of critical phenomena. Proc Natl Acad Sci USA 108:11361-11364

Varotsos PA, Sarlis NV, Skordas ES (2011c) Natural time analysis: the new view of time. Precursory seismic electric signals, earthquakes and other complex time-series. Springer, Berlin

Varotsos PA, Sarlis NV, Skordas ES, Lazaridou MS (2013) Seismic electric signals: an additional fact showing their physical interconnection with seismicity. Tectonophysics 589:116-125

Varotsos PA, Sarlis NV, Skordas ES (2016) On the motivation and foundation of natural time analysis: useful remarks. Acta Geophys 64:841-852

Zakharova O, Hainzl S, Bach C (2013) Seismic moment ratio of aftershocks with respect to main shocks. J Geophys Res Solid Earth 118:5856-5864

Zaliapin I, Ben-Zion Y (2013a) Earthquake clusters in southern California I: identification and stability. J Geophys Res Solid Earth 118:2847-2864

Zaliapin I, Ben-Zion Y (2013b) Earthquake clusters in southern California II: classification and relation to physical properties of the crust. J Geophys Res Solid Earth 118:2865-2877

Zaliapin I, Ben-Zion Y (2015) Artefacts of earthquake location errors and short-term incompleteness on seismicity clusters in southern California. Geophys J Int 202:1949-1968

Zaliapin I, Gabrielov A, Keilis-Borok V, Wong H (2008) Clustering analysis of seismicity and aftershock identification. Phys Rev Lett 101:018501 